\title{
BIO_SOS' EODHaM System Towards an Operational Habitat Monitoring Service
}

\author{
Jens STUTTE $^{1}$ and Stelios BOLLANOS ${ }^{2}$ \\ ${ }^{1}$ Planetek Italia s.r.1, Bari/Italy·stutte@planetek.it \\ ${ }^{2}$ Planetek Hellas EPE, Athens/Greece
}

This contribution was double-blind reviewed as extended abstract.

\begin{abstract}
The EODHaM system offers the right mix of site specific configurability and pre-engineered processing modules and workflows, which allow to offer a habitat monitoring service, particularly suited to support the multi-annual monitoring of Natura 2000 sites. The service provisioning foresees for each site an inception phase, during which the service provider collaborates with domain experts from the site in order to setup together the optimal service chain and processing configuration, customizing the workflow steps from the range of prebuilt, configurable processing modules.
\end{abstract}

\section{Introduction}

The FP7 project BIO_SOS elaborates the EODHaM (EO Data for Habitat Monitoring) System, which combines several stages of elaboration into a single workflow. The EODHaM system is an operational prototype of an ecological modelling system for effectively and multi-annual monitoring of Natura 2000 sites (and other ecologically sensitive sites) and their surrounding areas.

Due to several national and regional differences in policies/funding and the lack of a centralised management of biodiversity data, even at the same regional-local level, a noticeable effort is required to initiated a continuous, operational and quasi real-time monitoring of ecologically sensitive areas. In search of such a monitoring system, the starting point should be to know the main actors' requirements and expectations. According to them, it is expected that such a system should:

- function at fine spatial scales (1:5,000 or finer) where habitats ought to be represented,

- be user-oriented efficient and reliable,

- be sensitive to changes in the input datasets and the user-defined parameters,

- minimize the time between data acquisition and product delivery,

- minimize the involved costs (e.g., by reducing manpower, exploiting open source software solutions, etc.). 


\section{EODHaM System Definition}

The EODHaM system is defined in the BIO_SOS Architecture Design Document as a complete automatic system of workflow administration. The system will serve in efficiency the aim of the project for a multi-annual monitoring of Natura 2000 sites and their surrounding areas, as other ecologically sensitive sites. The EODHaM system will use different technologies, all well structured between them, with the final aim of processing, producing, cataloguing, and distributing the BIO_SOS products. The basic cornerstones and requirements of the system architecture can be summarized as follows:

- ability to integrate heterogeneous processing technologies,

- harmonization of single processors on external interface level through the usage of ISO Metadata for input descriptions,

- allow for the distribution of the system among several sites,

- clearly separate the (scientific) processor specific competences from the overall usage of the system.

The EODHaM system has been designed as a Service-Oriented Architecture (SOA). The SOA permits system developing in the form of interoperable web-based services. For that purpose the EODHaM system shall make use of the Web Services Business Process Execution Language (WS-BPEL), a standard executable XML-based language for specifying actions within business processes with web service. The WS-BPEL serves also as standardization of interaction between business processes in a distributed environment. A BPEL business process, for the exportation and importation of information, uses exclusively web service interfaces.

A common macro-workflow has been elaborated in the BIO_SOS Service Design Document, which is divided in three stages (Fig. 1). This macro-workflow guided the definition of the single processing modules' scope and common interfaces:

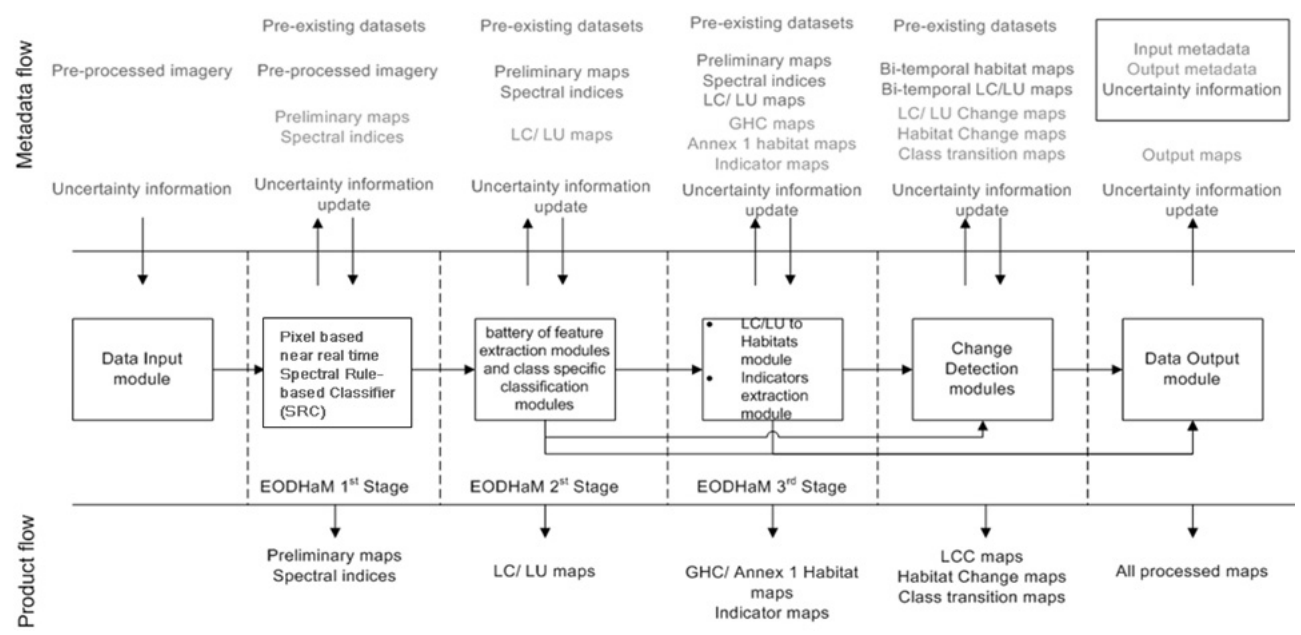

Fig. 1: EODHaM overall workflow 
The information flow between processing modules is based on the exchange of collections of ISO 19139 metadata records that describe the input files and their location. This allows a common interface definition for all processing modules. The execution of the EODHaM Business Process Workflow permits the interaction of all processor units and their related structured activities.

\section{Towards a BIO_SOS Service Definition}

During the elaboration of the system architecture, and due to the varying nature of the Nature 2000 sites the system shall be able to work on, a high need for site ("domain") specific customizations both of single processing steps and of entire workflow chains emerged. The use case analysis hence started from a system-external one: The definition of a new, domain specific chain.
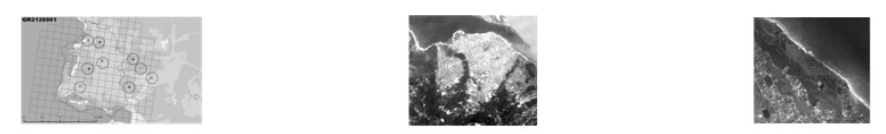

Domain

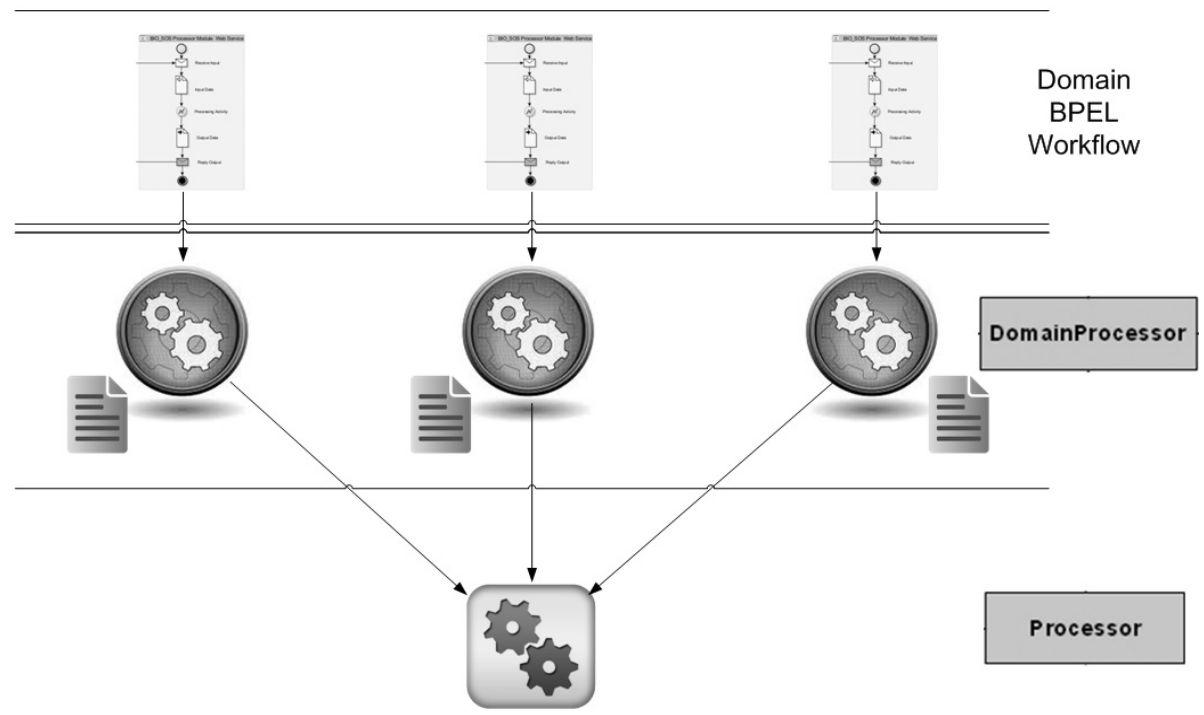

Fig. 2: Domain Processor encloses domain specific configurations

In terms of service definition, this adds an "Inception phase" to the service offer, during which the service provider offers to the customer a customization of the workflow for the specific site, defining a set of parameters, choosing ancillary data and configuring the processing modules with domain specific configurations. The complexity of this "Inception phase" depends on the product's complexity itself (different BIO_SOS products have different levels of elaboration) and the given, site-specific constraints. The "Inception phase" 
needs collaboration and input from the domain experts (mostly scientists) that created the theoretical basis for the involved processing modules.

So for many use cases, the BIO_SOS monitoring service offering cannot be seen as an outof-the-box solution, but the highly configurable EODHaM system together with the conceptual separation of the main processing steps enable BIO_SOS to offer a highly specialized service based on a proven-to-work system and a well-defined set of product types the system can deliver.

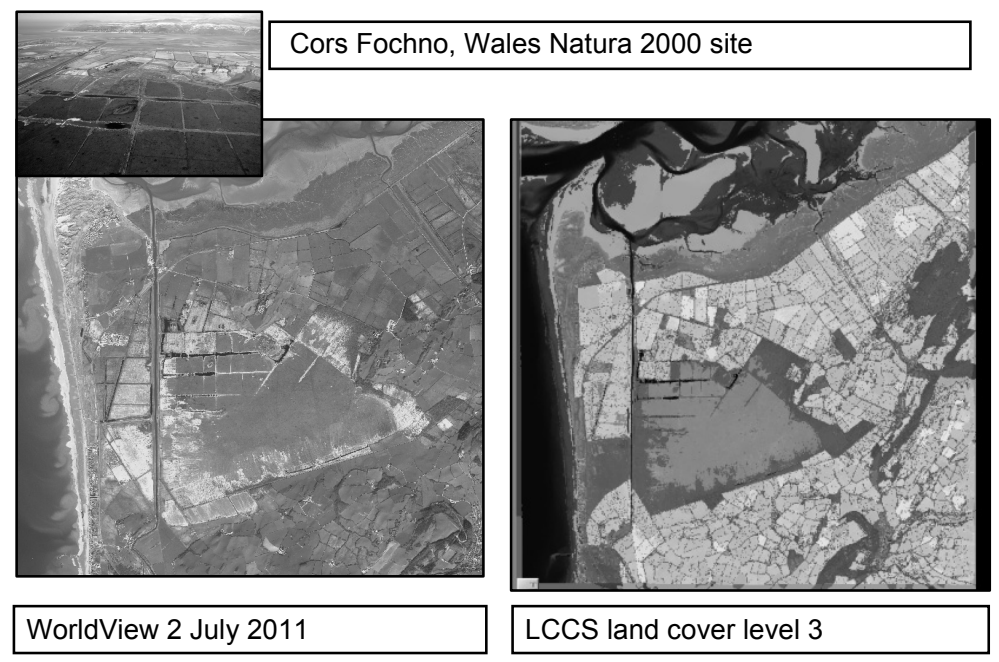

Fig. 3: LCCS classification of Cors Fochno, Wales

A first example of customization has already been done on the Natura 2000 site Cors Fochno (Wales). Starting from satellite images acquired in July 2011 by WorldView 2 and ancillary data, a classification of the site has been done trough the definition of site specific parameters for the classification rules to identify the habitat classes. 